\title{
Valor social generado por una entidad de integración sociolaboral: un estudio de caso
}

Social value generated by a socio-labour integration entity: a case study

\begin{abstract}
Resumen
El objetivo del presente trabajo es analizar el impacto social de una entidad dedicada a la integración sociolaboral de personas con discapacidad desde una perspectiva de contabilidad social, calculando el Valor Social Integrado (VSI). El estudio muestra la aplicación de esta metodología al caso de La Fundació Rubricatus. El análisis ha seguido un proceso de investigación-acción participativa con representantes de la entidad y sus grupos de interés (personas trabajadoras y usuarias con discapacidad, sus familias, Administraciones públicas, etc.), lo que ha permitido identificar de manera sistemática las dimensiones o variables de valor percibidas como relevantes. Como resultado final se calcula el VSI, que expresa en términos monetarios el valor social generado por la entidad, tanto el valor ligado a su actividad económica como los beneficios creados más allá de las transacciones económicas. EI VSI permite a las entidades de apoyo a las personas con discapacidad demostrar su valor social añadido.
\end{abstract}

\section{Palabras clave}

Discapacidad, integración sociolaboral, valor social, contabilidad social, impacto social.

\begin{abstract}
The aim of this paper is to analyse the social impact of an entity dedicated to socio-labour integration of disabled persons from a social accounting perspective, calculating the Integrated Social Value (VSI). The study shows the application of this methodology to the case of the Rubricatus Foundation. The analysis has followed a participatory action research process with representatives of the entity and its stakeholders (workers and users with disabilities, their families, public administrations, etc.), which has allowed to identify systematically the value dimensions or variables perceived as relevant. As a final result, the VSI is calculated, which expresses in monetary terms the social value generated by the entity, both the value linked to its economic activity and the benefits created beyond economic transactions. The VSI allows entities that support people with disabilities to demonstrate their social added value.
\end{abstract}

\section{Keywords}

Disability, socio-labour integration, social value, social accounting, social impact.

\author{
Silvia Ayuso Siart \\ <silvia.ayuso@esci.upf.edu>
}

ESCI-UPF. Universitat Pompeu Fabra. España

\section{Jordi García Castillo}

<jgarcia@rubricatus.org>

La Fundació Rubricatus. España

Immaculada Pérez Canillo <iperez@rubricatus.org>

La Fundació Rubricatus. España

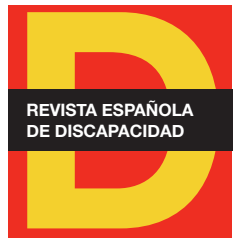

Para citar:

Ayuso, S. et al. (2020). "Valor social generado por una entidad de integración sociolaboral: un estudio de caso". Revista Española de Discapacidad, 8(2), pp. 107-127.

Doi: <https://doi.org/10.5569/23405104.08.02.05>

Fecha de recepción: 17-04-2020 Fecha de aceptación: 05-10-2020 


\section{Introducción}

Los centros especiales de empleo y centros ocupacionales juegan un papel clave en la integración social y laboral de las personas con discapacidad, por lo que su impacto para la sociedad es evidente. Sin embargo, los intentos de evaluar el impacto social de las entidades dedicadas a la integración sociolaboral de personas con algún tipo de discapacidad se han limitado mayoritariamente a estimar los costes (y beneficios) económicos para la sociedad (por ejemplo, Jordán de Urríes et al., 2014; Retolaza, 2012; Rodríguez et al., 2015). Con la intención de medir el valor social de diferentes iniciativas de atención a la discapacidad, se han realizado algunos estudios de Retorno Social de la Inversión (Social Return on Investment, SROI por sus siglas en inglés) ${ }^{1}$. En España, esta metodología se ha aplicado para medir el impacto de la asistencia personal en Barcelona (Ayuntamiento de Barcelona, 2012), comparar la asistencia personal y residencial en Madrid (Huete y Quezada, 2014), analizar el retorno social de la inversión pública en varios centros especiales de empleo y centros ocupacionales en distintas comunidades autónomas (por ejemplo, ECODES y Alter Civites, 2013; ECODES y Alter Civites, 2018) y medir el impacto económico y social del proyecto de servicio de asistencia personal en Andalucía (láñez et al., 2018).

No obstante, la metodología SROI sufre de algunas limitaciones relacionadas con la subjetividad inherente en establecer el efecto causado por el proyecto analizado y los elevados recursos para llevar a cabo la evaluación (Maier et al., 2015). Asimismo, los estudios realizados centran su análisis en el impacto resultante de la intervención social, y generalmente dejan de lado otros beneficios experimentados por los grupos de interés que se interrelacionan con el proyecto o la organización, como personal técnico, personas voluntarias, estudiantes en prácticas, proveedores, ciudadanía, etc. (Ayuso, 2020).

En este sentido, resulta interesante analizar el impacto social de una entidad dedicada a la integración sociolaboral de personas con discapacidad desde una perspectiva de contabilidad social, calculando el Valor Social Integrado (VSI). La metodología del VSI permite objetivar y expresar en términos monetarios el valor generado por una organización, integrando los resultados económicos y sociales en un único concepto de generación de valor. El interés de este modelo de contabilidad social recientemente propuesto consiste en su aproximación multidimensional o multistakeholder: el valor creado no se establece de una única forma sino como el conjunto de percepciones de los grupos de interés o stakeholders (Retolaza et al., 2016). Asimismo, el hecho de asignar un valor monetario al impacto social permite que se integre con el impacto económico de la organización analizada y se transforme en indicadores útiles para la comunicación externa y la gestión interna.

El objetivo del presente trabajo es calcular el VSI de una entidad dedicada a la promoción de la calidad de vida de las personas con discapacidad intelectual. A continuación, presentamos la entidad del estudio de caso, La Fundació Rubricatus y describimos la metodología aplicada. Posteriormente presentamos los resultados del análisis cualitativo (identificación de los grupos de interés y del valor que perciben) y del análisis cuantitativo (monetización del valor) realizado. Finalmente, cerramos el trabajo con las conclusiones que se derivan del estudio.

1. El concepto de valor social hace referencia a una forma de cuantificar la importancia relativa de aquellos cambios en las vidas de las personas que no están reflejados en transacciones financieras o de mercado (Social Value International, 2019). 


\section{La Fundació Rubricatus}

La Fundació Rubricatus se constituyó el 12 de junio de 1997. La entidad representa la culminación de una meritoria labor de un grupo de ciudadanos del Prat de Llobregat (Barcelona), la mayoría padres y madres con hijos con discapacidad intelectual, que desde los años 70 y con el fin de ofrecer alternativas educativas y laborales a sus hijos, fundaron la Escuela de Educación Especial Sant Isidre, hoy el Centro de Educación Especial Can Rigol, y el antiguo Taller Especial Sant Isidre, precursor del actual Centro Especial de Trabajo Tesiprat Serveis. Este hecho representó un éxito sin precedentes en la lucha contra la exclusión social de las personas con discapacidad intelectual en el municipio. Más adelante, y gracias a la implicación y el compromiso de toda la ciudad mediante el Ayuntamiento del Prat de Llobregat, que en ese momento gestionaba el antiguo Patronato Municipal de Ayuda al Disminuido, se acordó crear una única entidad que diera respuesta a las diferentes necesidades de las personas con discapacidad intelectual.

Hoy en día, La Fundació Rubricatus es una entidad de iniciativa social y sin ánimo de lucro, que tiene como finalidad lograr la máxima calidad de vida de las personas adultas con discapacidad intelectual del Prat de Llobregat, contribuyendo a satisfacer sus necesidades vitales a través de soluciones sociolaborales y de empoderamiento personal alineadas con sus capacidades y diversidad funcional. Tiene como uno de sus objetivos principales promover la integración social efectiva de las personas con discapacidad, ya sea a través de la prestación de servicios de terapia ocupacional, apoyando su actividad profesional o mediante el apoyo a las familias de las personas trabajadoras o usuarias. Para alcanzar su misión, La Fundació Rubricatus cuenta con los siguientes servicios de apoyo:

- Centro Especial de Empleo (CEE) Tesiprat: El CEE lleva a cabo actividades empresariales de mercado y presta servicios diversos de apoyo a la actividad profesional de las personas con discapacidad que tiene contratadas en las diferentes secciones productivas, como la sección de Catering Ésbo o el bar/ cafetería Sagnier. Tesiprat Servicios utiliza una estrategia de diversificación en su oferta de servicios, la formación profesional en el puesto de trabajo y los servicios de apoyo a la actividad profesional, todo ello en un entorno laboral real, como herramientas para la creación de puestos de trabajo y la inserción laboral de las personas con discapacidad.

- Servicio de Terapia Ocupacional (STO): El STO es un servicio que está en funcionamiento desde la década de 1970 y es la alternativa dentro del ámbito de los servicios sociales para la integración de aquellas personas que no pueden incorporarse al sistema ordinario de trabajo ni a un CEE, porque no disponen de una capacidad productiva suficiente para establecer una relación laboral contractual.

- Servicio Ocupacional de Inserción (SOI): El SOI comienza a funcionar en 1996 como un servicio ocupacional diurno de tipo transitorio y formativo, que atiende a personas con discapacidad intelectual con perfil productivo pero que aún no están en condiciones de integrarse a la dinámica laboral por diferentes motivos.

- Servicio de Apoyo Familiar: El Servicio de Apoyo Familiar da respuesta a la necesidad de compartir un espacio con otras familias que se encuentran en una situación similar, a fin de poder intercambiar experiencias, información y resolver dudas sobre el mundo de la discapacidad intelectual. 
Desde el año 2016, La Fundació Rubricatus obtiene la mayoría de sus ingresos por la prestación de servicios, aunque sigue contando con una aportación importante de subvenciones. La tabla 1 muestra las cifras de personas contratadas y atendidas en el último año.

\begin{tabular}{l}
\hline Tabla 1. Cifras de actividad de La Fundació Rubricatus (año 2019) \\
\begin{tabular}{|l|l|}
\hline Personas con discapacidad acompañadas (trabajadoras y usuarias) & 175 \\
\hline Personas contratadas con discapacidad & 100 \\
\hline Personas usuarias de los Servicios Ocupacionales & 75 \\
\hline Familias acompañadas & 160 \\
\hline
\end{tabular}
\end{tabular}

Fuente: datos de La Fundació Rubricatus a 31/12/2019.

\section{Metodología aplicada}

La metodología del análisis del Valor Social Integrado (VSI), desarrollada por Retolaza et al. (2014), es un sistema de contabilidad social que permite cuantificar y monetizar el valor creado por la actividad económica y/o social de una organización. La metodología se fundamenta en la perspectiva de la teoría de los grupos de interés o stakeholders (Freeman, 1984; Freeman et al., 2010). Desde esta perspectiva, se plantea considerar un concepto más amplio del valor creado por una organización -por un lado, incluyendo el valor distribuido a todos los stakeholders de la organización y no sólo a sus propietarios o accionistas (shareholders), y por otro lado, incorporando los efectos no económicos sobre los diversos stakeholders-. Esto da lugar a una visión más completa del valor generado, a la que se le puede denominar triple cuenta de resultados (triple bottom line) (Elkington, 1997), blended value (Emerson et al., 2003), valor compartido (shared value) (Porter y Kramer, 2011) o Valor Social Integrado.

El sistema de contabilidad social propuesto se basa en el llamado modelo poliédrico, que permite objetivar y visualizar el valor que crea una organización para el conjunto de sus grupos de interés (Retolaza et al., 2016). El modelo poliédrico se muestra gráficamente en la figura 1. Las diferentes áreas sombreadas en gris representan el valor social (social value, SV) creado para cada stakeholder (S). Mientras que el círculo interior sombreado en azul claro representa el valor compartido por todos los grupos de interés, los "pétalos" exteriores sombreados en azul oscuro representan los valores generados para grupos de interés determinados que no coinciden con los de otros grupos. El valor total generado por la organización corresponde al área total sombreada. 
Figura 1. Modelo poliédrico de análisis del Valor Social Integrado

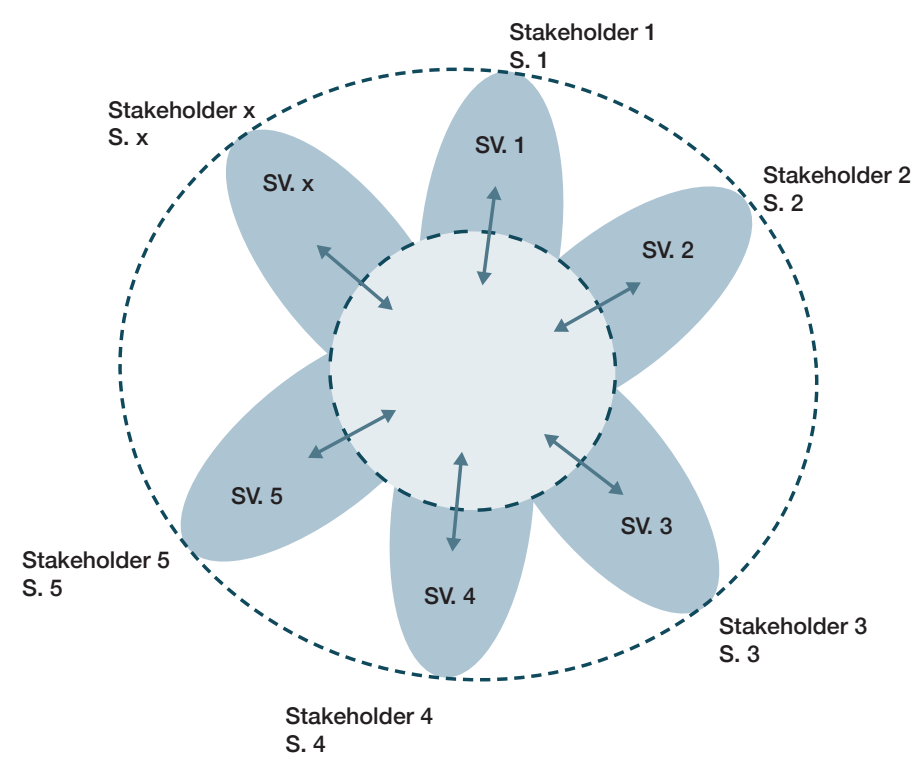

Fuente: Retolaza et al. (2016).

EI VSI se compone del valor social generado a través de la actividad económica o mercantil -captado por indicadores de contabilidad - y el valor social específico que la organización genera para sus diversos grupos de interés mediante relaciones ajenas a las transacciones de mercado. Para determinar el valor social específico o valor de no mercado se combina un análisis cualitativo y cuantitativo. El análisis cualitativo busca identificar el valor que la organización genera para sus grupos de interés más allá de la actividad económica, basándose en un proceso de consulta con representantes de los stakeholders. El análisis cuantitativo se centra en cuantificar el valor percibido a través del desarrollo de indicadores cuantificables vinculados a proxies (aproximaciones) financieras, que permitan monetizar el valor generado. Las fases del proceso de análisis del VSI se muestran en la figura 2.

Figura 2. Fases del análisis del Valor Social Integrado

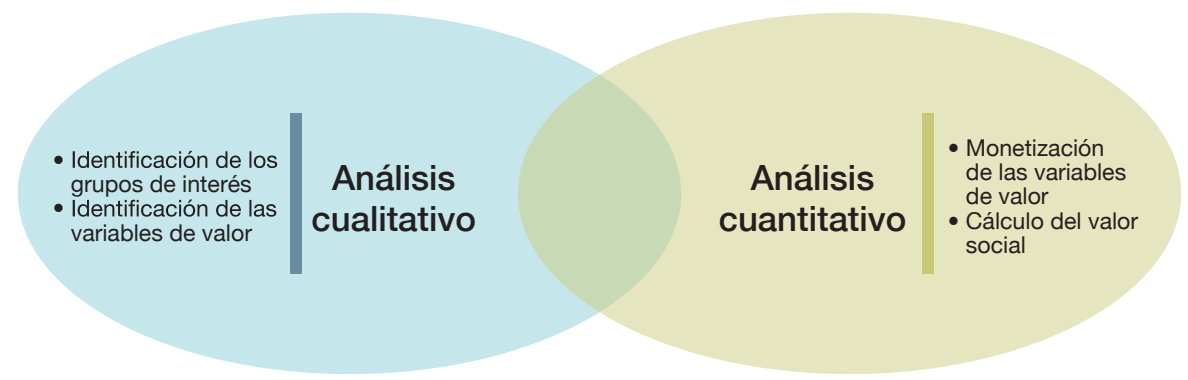

Fuente: elaboración propia. 
La contabilidad social del VSI se ha aplicado a más de 50 organizaciones de diferentes tamaños y sectores, tanto lucrativas como sin ánimo de lucro (Retolaza y San-José, 2018). Debido a las características del enfoque, en cada caso se considera el contexto específico de la organización en cuestión y se sigue un proceso de "investigación-acción" (action research) en estrecha colaboración con los agentes pertenecientes a la organización analizada y sus grupos de interés. Como otras metodologías de medición de impacto social, el VSI puede presentar dificultades en relación con la disponibilidad de los datos necesarios para la cuantificación del valor y el proceso de monetización (encontrar valores sustitutivos adecuados) (Maier et al., 2015). Sin embargo, a diferencia del enfoque del SROI que aspira a determinar los cambios significativos experimentados por los principales stakeholders afectados, el VSI se conforma con medir el valor de los productos y servicios tangibles resultantes de las actividades de la organización analizada. Así, mientras los estudios de SROI dedican un esfuerzo considerable a la recogida de datos primarios para evaluar los cambios sucedidos y adoptan una serie de supuestos para aislar el efecto causado por la actividad analizada, el análisis del VSI se centra en los efectos directamente atribuibles a la organización que aportan valor al stakeholder en el período temporal de referencia.

En los siguientes apartados se presentan los resultados de los análisis cualitativo y cuantitativo aplicados al caso de La Fundació Rubricatus. El alcance del análisis comprende todas las actividades realizadas por la fundación, tanto del Centro Especial de Empleo como del Servicio de Terapia Ocupacional, del Servicio Ocupacional de Inserción y del Servicio de Apoyo Familiar. El período temporal considerado para la contabilidad social es el último ejercicio fiscal con datos disponibles en el momento del estudio, el año 2018.

\section{Análisis cualitativo: la identificación de los stakeholders y el valor percibido}

El análisis del Valor Social Integrado (VSI) parte de la premisa de que el resultado de una actividad sólo se transforma en valor cuando hay alguien para valorarlo, por lo que adopta una aproximación fenomenológica al análisis. Esto quiere decir que el fenómeno del valor social se aborda a través de las percepciones de las personas que reciben este valor, es decir, de las diferentes partes interesadas de la organización (stakeholders). En lugar de definir las dimensiones de valor basándose en una categorización preliminar del bien social, el investigador aborda el fenómeno sin criterios interpretativos previos y permite a los propios grupos de interés expresarse sobre la generación de valor que perciben de la organización.

Así, como primer paso se identificaron los stakeholders a los que La Fundació Rubricatus presupone que genera algún tipo de valor, ya sea monetario o no monetario (por ejemplo, vinculado a la mejora de competencias o de bienestar). La tabla 2 ofrece una breve descripción de cada grupo de interés identificado, e ilustra los diferentes tipos de relaciones que mantiene la fundación con sus stakeholders y que dan lugar a diferentes perspectivas de creación de valor social. 


\begin{tabular}{|c|c|}
\hline Grupo de interés & Descripción \\
\hline $\begin{array}{l}\text { Personas trabajadoras con } \\
\text { discapacidad }\end{array}$ & Personas trabajadoras con discapacidad con contrato laboral \\
\hline Personas usuarias & Personas con discapacidad atendidas a través de los Servicios Ocupacionales \\
\hline $\begin{array}{l}\text { Familias de personas } \\
\text { trabajadoras o usuarias }\end{array}$ & Familias de personas trabajadoras y usuarias con discapacidad \\
\hline Personal técnico & Personas trabajadoras con contrato laboral sin certificado de discapacidad \\
\hline Socios/as y donantes & $\begin{array}{l}\text { Personas o entidades que de forma individual o corporativa hacen aportaciones } \\
\text { económicas voluntarias a la entidad para poder afrontar inversiones y poner en marcha } \\
\text { proyectos y actividades }\end{array}$ \\
\hline Estudiantes en prácticas & Estudiantes de diferentes universidades y ciclos formativos que realizan prácticas \\
\hline Voluntarios/as & $\begin{array}{l}\text { Personas que de forma individual o a través de sus empresas, aportan conocimiento, } \\
\text { tiempo y experiencia para llevar a cabo actividades regulares así como acciones } \\
\text { puntuales }\end{array}$ \\
\hline $\begin{array}{l}\text { Entidades de servicios básicos } \\
\text { de atención a la persona }\end{array}$ & $\begin{array}{l}\text { Red de servicios que complementan las diversas necesidades de las personas con } \\
\text { diversidad intelectual (servicios sociales, servicios de salud, centros de atención } \\
\text { primaria, servicios especializados en salud mental, etc.) }\end{array}$ \\
\hline $\begin{array}{l}\text { Entidades colaboradoras en el } \\
\text { ámbito de la discapacidad }\end{array}$ & Red de entidades en torno a las personas con discapacidad de ámbito local y supralocal \\
\hline Otras entidades de ámbito local & Entidades locales de la economía social que interactúan con La Fundació Rubricatus \\
\hline Administraciones públicas & Ayuntamiento del Prat de Llobregat y resto de administraciones \\
\hline Clientes & $\begin{array}{l}\text { Clientes que contratan los servicios del Centro Especial de Empleo (jardinería, limpieza y } \\
\text { mantenimiento, catering, hostelería, etc.) }\end{array}$ \\
\hline Proveedores & Proveedores tanto de servicios como de suministros \\
\hline Ciudadanía & $\begin{array}{l}\text { Personas que tienen una relación directa o indirecta con La Fundació Rubricatus } \\
\text { (vecinos, Amigos de La Fundació, escuelas del municipio, personas seguidoras en las } \\
\text { redes sociales, etc.) }\end{array}$ \\
\hline
\end{tabular}

Fuente: elaboración propia.

El siguiente paso fue identificar los principales aspectos en los que La Fundació Rubricatus crea valor para los diferentes stakeholders, las llamadas "variables de valor", a través de un proceso de consulta. Con esta finalidad se realizaron una serie de entrevistas en profundidad a diferentes interlocutores como representantes de los stakeholders perceptores del valor ${ }^{2}$. En total se llevaron a cabo 26 entrevistas individuales a distintos miembros del equipo directivo y del equipo técnico de administración, así como a seleccionados miembros del patronato que pudiesen aportar la visión de grupos de interés externos a la fundación. Además, se pasó un cuestionario a todas las personas usuarias del Centro Ocupacional, a las familias, a las personas trabajadoras, al personal técnico y a las personas voluntarias, que fue contestado por 85 personas. La información recogida en las entrevistas y los cuestionarios se analizó siguiendo los pasos del enfoque fenomenológico: delimitación de las unidades de significado expresadas, depuración del lenguaje coloquial e identificación de las ideas subyacentes. Finalmente, estas ideas relacionadas con las diferentes

2. A la hora de seleccionar los interlocutores de los stakeholders no se trata de diseñar una muestra representativa según criterios estadísticos. Con la intención de realizar un muestreo cualitativo, se buscan interlocutores que sean accesibles y que tengan capacidad para aportar información rica y completa. 
dimensiones de valor percibidas por los grupos de interés se reestructuraron en términos que permitieran una medición. Se celebraron tres reuniones grupales con las familias, el equipo técnico y las personas trabajadoras para contrastar y validar las "variables de valor" identificadas.

Como resultado se obtuvo un conjunto de 12 variables de valor potencialmente cuantificables, que pueden relacionarse con cada uno de los grupos de stakeholders en una matriz de variables de valor. La tabla 3 muestra esta matriz, en la que aparecen solamente los colectivos para los cuales se identificó alguna variable de valor particular: personas trabajadoras con discapacidad, personas usuarias, familias de personas trabajadoras o usuarias, personal técnico (trabajadores/as sin discapacidad), estudiantes en prácticas, voluntarios/as, Administraciones públicas (AAPP), clientes, y ciudadanía. La visión del resto de los grupos de interés identificados previamente (tabla 2) se ha englobado en la perspectiva de las AAPP o de la ciudadanía como stakeholder receptor de valores transversales, como por ejemplo la sensibilización o el impacto medioambiental.

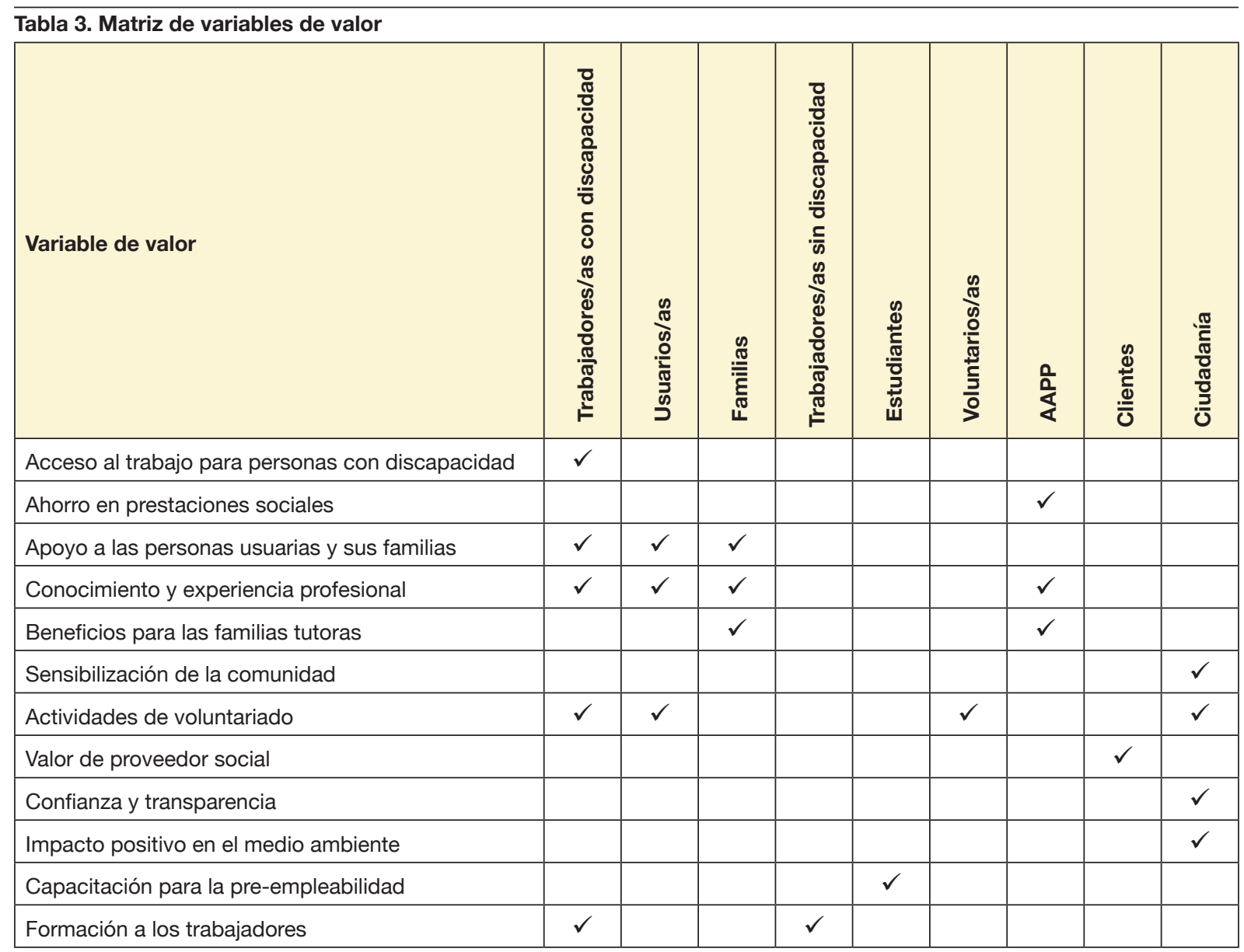

Fuente: elaboración propia. 
Por otra parte, se identificaron una serie de aspectos relacionados con la generación de valor emocional, más difícil de cuantificar y monetizar, pero que también forman parte de la percepción del valor aportado por la fundación. La tabla 4 muestra estas variables de valor intangibles que son percibidas por las personas trabajadoras y usuarias con discapacidad y por los colectivos con relación directa con éstos (personal técnico, familias, voluntarios y estudiantes en prácticas).

Tabla 4. Variables de valor emocional
\begin{tabular}{|l|l|}
\hline Bienestar personal & $\begin{array}{l}\text { Ilusión, crecimiento personal, paciencia, capacidad de relativizar, experiencia } \\
\text { vital, mejora de la autoestima, estabilidad emocional, tranquilidad, motivación, } \\
\text { capacidad y posibilidad de decisión. }\end{array}$ \\
\hline Relaciones sociales & $\begin{array}{l}\text { Relaciones con familias, compañeros/as, usuarios/as etc.: en condiciones de } \\
\text { pleno derecho, pasar a ser sujetos activos y visibles. }\end{array}$ \\
\hline Identidad comunitaria & $\begin{array}{l}\text { Formar parte de un grupo con unos objetivos comunes, y en consecuencia, dejar } \\
\text { de sentirse sólo, aislado y diferente. }\end{array}$ \\
\hline Compañerismo & Relaciones personales: dar apoyo y recibirlo. \\
\hline Respeto hacia el trabajo realizado & Mejora de la autoestima y de la autoconfianza. \\
\hline Reconocimiento & $\begin{array}{l}\text { Agradecimiento por el trabajo hecho, tanto por parte de familiares, ciudadanía, } \\
\text { resto de profesionales, etc. }\end{array}$ \\
\hline Ser un referente & $\begin{array}{l}\text { Consideración de un referente para otros (p.ej. hijos de trabajadores/as) y para } \\
\text { otras personas con discapacidad que todavía no han alcanzado este bienestar. }\end{array}$ \\
\hline Responsabilidad & Responsabilidad añadida de trabajar con colectivo de personas con discapacidad. \\
\hline
\end{tabular}

Fuente: elaboración propia.

\section{Análisis cuantitativo: la monetización del valor}

El Valor Social Integrado (VSI) consta de dos componentes, el valor social de la actividad económica o valor social de mercado, y el valor social específico o valor de no mercado (ver figura 3). El primero de ellos hace referencia al valor social que la organización genera con la realización de su actividad económica, siendo transferido como contrapartida de un precio real de mercado (y recogido en la contabilidad de la organización). El segundo de los componentes del VSI, el valor social específico o de no mercado, es concebido como el valor transferido por la organización a sus grupos de interés sin contrapartida de un precio real de mercado. 
Figura 3. Componentes del Valor Social Integrado (VSI)

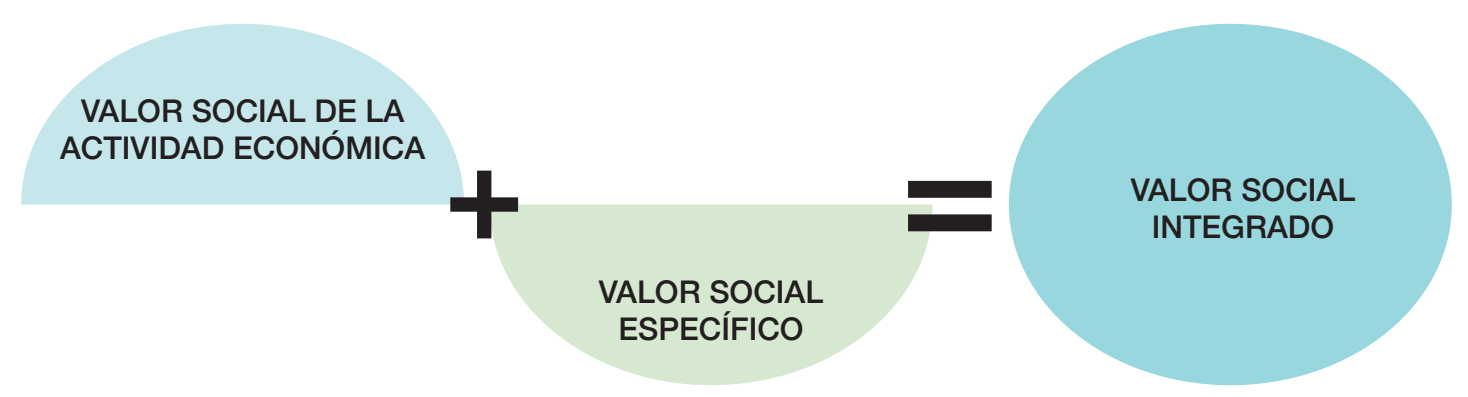

Fuente: elaboración propia.

\subsection{Cálculo del valor social generado por la actividad económica}

El valor social generado por la actividad económica de una organización (también llamado valor socioeconómico o valor económico social, VES) representa la creación y distribución de riqueza para los grupos de interés. Mientras que la cuenta de resultados tradicional equipara riqueza con el resultado económico obtenido por la organización, el VES toma una perspectiva más amplia y, además del excedente económico, considera la riqueza creada para los diferentes grupos de interés que se relacionan con la actividad económica de la organización, como son organizaciones proveedoras, personas trabajadoras y Administraciones públicas.

Puede considerarse como valor económico social directo el valor añadido que una organización genera y distribuye, y como valor económico social indirecto el valor añadido que genera con la compra de recursos externos. El valor económico social directo (VES-D) se compone entonces del valor económico distribuido a las personas trabajadoras (salarios netos), a las Administraciones públicas (cotizaciones a la seguridad social, impuesto sobre la renta, IVA y otros impuestos abonados) y el valor económico distribuido y retenido en la propia organización (resultado económico y amortizaciones) ${ }^{3}$. La tabla 5 muestra estos valores para La Fundació Rubricatus en el año 2018.

\begin{tabular}{l}
\hline Tabla 5. Generación y distribución de valor económico social de La Fundació Rubricatus \\
\begin{tabular}{|l|c|}
\hline Valor económico social directo (VES-D) & $\mathbf{2 0 1 8}$ \\
\hline Valor distribuido a personas trabajadoras & $2.136 .757,71 €$ \\
\hline Valor distribuido a Administraciones públicas & $623.171,42 €$ \\
\hline Valor retenido en la organización & $74.698,51 €$ \\
\hline TOTAL & $\mathbf{2 . 8 3 4 . 6 2 7 , 6 4 €}$ \\
\hline
\end{tabular}
\end{tabular}

Fuente: elaboración propia.

3. En este caso se consideran despreciables los gastos financieros como valor distribuido a los proveedores financieros. 
El valor económico social indirecto (VES-I) corresponde al efecto tractor de la compra efectuada a los proveedores de bienes y servicios de primer nivel, es decir, al valor económico movilizado a través de la compra. Con el fin de no incluir como valor social el consumo de materias primas y energía, sólo se tiene en cuenta el valor añadido por los proveedores de manera proporcional al volumen de compras efectuado.

Para el cálculo del VES-I se realizó un análisis de la estructura de la distribución de valor de los proveedores de la fundación, consultando sus datos mercantiles en la base de datos SABI (Sistema de Análisis de Balances Ibéricos). De este modo, se obtuvieron unos índices de repercusión, expresados como ratios de facturación, que sirvieron de proxy para estimar la distribución de valor de los proveedores. A partir de estos índices de repercusión se imputó el porcentaje que los proveedores distribuyen a sus trabajadores/as, a las Administraciones públicas, y el valor económico distribuido y retenido en las propias organizaciones proveedoras. La tabla 6 muestra el valor económico movilizado por La Fundació Rubricatus en el año 2018, diferenciando el valor distribuido a los propios proveedores (trabajadores/as e inversores) y el valor distribuido a las Administraciones públicas vía impuestos.

\begin{tabular}{l}
\hline Tabla 6. Movilización de valor económico social de La Fundació Rubricatus \\
\begin{tabular}{|l|c|}
\hline Valor económico social indirecto (VES-I) & $\mathbf{2 0 1 8}$ \\
\hline Valor movilizado y distribuido a proveedores & $120.693,82 €$ \\
\hline Valor movilizado y distribuido a Administraciones públicas & $74.603,60 €$ \\
\hline TOTAL & $\mathbf{1 9 5 . 2 9 7 , 4 2} €$ \\
\hline
\end{tabular}
\end{tabular}

Fuente: elaboración propia.

Junto al valor distribuido a personas trabajadoras, proveedores y Administraciones públicas también se puede considerar el valor distribuido a los clientes (VES-C) de la organización y equiparable al importe de las ventas de los bienes y servicios. En el caso de La Fundació Rubricatus, la prestación de servicios a través de las diversas actividades empresariales de mercado generó una facturación de 1.806.401,90 € en el ejercicio fiscal considerado. El valor económico social total (VES-D + VES-I+VES-C) generado por La Fundació Rubricatus en el año 2018 asciende a 4.836.326,96 €.

\subsection{Cuantificación y monetización del valor social específico}

El valor social específico (VASE) se refiere al valor de no mercado que la organización distribuye entre sus grupos de interés, e integra el conjunto de variables de valor identificadas en la fase cualitativa de consulta con los stakeholders (tabla 3). Con el objetivo de cuantificar y expresar en términos monetarios las variables de valor, se identificaron uno o más indicadores relacionados con cada una de estas variables, que fueran capaces de medir los productos y servicios tangibles resultantes de las actividades realizadas por la fundación (outputs) y cuya información estuviera disponible, como por ejemplo el número de personas trabajadoras con discapacidad o el número de horas de voluntariado. Para estimar el valor monetario de estos outputs que no son objeto de comercialización, se identificaron posibles aproximaciones al valor o proxies financieras. La selección de proxies se basó en la consideración de precios de mercado de los 
outputs suministrados por la fundación o de servicios alternativos equiparables, en la cuantificación de los costes evitados gracias a los servicios prestados por la fundación y en valoraciones realizadas por la Administración Pública (por ejemplo, subvenciones). En la medida de lo posible, los indicadores y las proxies elegidas - siempre conservadoras - fueron consensuados con representantes de otras entidades de la economía social del municipio, que emprendieron el cálculo del VSI en paralelo con La Fundació Rubricatus. En aquellos casos en los que no fue posible identificar una proxy adecuada, se utilizó el coste realizado o estimado, suponiendo que el valor generado se corresponde como mínimo con el gasto incurrido.

Finalmente, se determinaron unos algoritmos de cálculo que relacionaran los outputs con las proxies para cuantificar el valor monetario de cada variable de valor para el período de referencia. El resultado final es una tabla de valoración en la que se han monetizado los aspectos en los que la organización genera valor social a los diferentes stakeholders. La tabla 7 muestra los indicadores y proxies utilizados para el cálculo, y los valores monetarios resultantes para las variables de valor. Mientras en algunas variables, el valor se genera para un grupo de interés específico, en otras variables se genera de forma simultánea -como valor compartido - para varios stakeholders (apoyo a las personas usuarias y sus familias, conocimiento y experiencia profesional, beneficios para las familias tutoras, actividades de voluntariado y formación a los trabajadores/as) ${ }^{4}$.

El valor social específico (VASE) generado por La Fundació Rubricatus se corresponde con la suma de las variables de valor monetizadas y asciende a 2.552.568,72 € para el año 2018. Aparte de la retribución económica recibida por las personas trabajadoras (con o sin discapacidad) ya considerada en el valor económico social $(2.137 \mathrm{k} €)$, la mayor aportación de valor se realiza a través del acceso al trabajo para personas con discapacidad (465 $\mathrm{k} €$ ) y los beneficios para las familias tutoras ( $1.061 \mathrm{k} €$ ), todos ellos aspectos estrechamente ligados a la misión de la fundación de lograr la máxima calidad de vida de las personas adultas con discapacidad intelectual del municipio. También son importantes la confianza y transparencia de la gestión de la fundación percibida (329 k €), el ahorro en prestaciones sociales (186 k €), el impacto positivo en el medio ambiente derivado de los proyectos realizados de economía circular (182 k €) y el conocimiento y experiencia profesional en los servicios prestados al colectivo de personas con discapacidad intelectual del Prat de Llobregat (153 k €). Otros efectos generados por La Fundació Rubricatus, como la sensibilización, el voluntariado o la formación, se traducen en menores cifras de valor monetario, debido al menor alcance de estas actuaciones.

Cabe señalar que no se cuantificaron las mejoras en el bienestar de las personas con discapacidad (trabajadoras y usuarias) debido a su carácter intangible. A pesar de que la fundación aplica diferentes sistemas de evaluación que evidencian la mejora de las capacidades de las personas con diversidad funcional trabajadoras o usuarias (por ejemplo, programa individual de intervención, evaluación de la conducta adaptativa, evaluación funcional o evaluación multimodal), resulta complejo identificar la aportación de valor atribuible a la actividad realizada por la organización. Por esta razón, la cifra obtenida del valor social específico puede considerarse una primera aproximación a este valor, que puede ser complementada en ejercicios futuros.

4. En los casos en los que el valor monetizado se genera para más de un stakeholder (personas trabajadoras y/o usuarias) pero no se trate de valor coincidente, se ha realizado una asignación del valor en función del número de personas integrantes de cada colectivo. 


\begin{tabular}{|c|c|c|c|c|}
\hline Variable de valor & Indicador & Proxy & Fuente & $\begin{array}{c}\text { Valor } \\
\text { monetario } 2018\end{array}$ \\
\hline $\begin{array}{l}\text { Acceso al trabajo } \\
\text { para personas con } \\
\text { discapacidad }\end{array}$ & $\begin{array}{l}\mathrm{N}^{\circ} \text { de trabajadores/as con } \\
\text { discapacidad (de especial } \\
\text { dificultad) }\end{array}$ & $\begin{array}{l}\text { Valor que la administración atribuye } \\
\text { anualmente por el mantenimiento } \\
\text { de un puesto de trabajo de } \\
\text { estas características (suma de } \\
\text { subvenciones) }\end{array}$ & $\begin{array}{l}\text { Departamento de Trabajo, } \\
\text { Asuntos Sociales y } \\
\text { Familias de la Generalitat } \\
\text { de Catalunya: Orden } \\
\text { TSF/91/2017, de } 15 \text { de } \\
\text { mayo }\end{array}$ & $465.493,42 €$ \\
\hline $\begin{array}{l}\text { Ahorro en } \\
\text { prestaciones sociales }\end{array}$ & $\begin{array}{l}N^{\circ} \text { de trabajadores/as CEE que no } \\
\text { reciben prestaciones públicas }\end{array}$ & $\begin{array}{l}\text { Cuantía de pensión no contributiva } \\
\text { de invalidez/complemento por } \\
\text { necesidad de tercera persona }\end{array}$ & $\begin{array}{l}\text { Datos del Departamento } \\
\text { de Trabajo, Asuntos } \\
\text { Sociales y Familias de la } \\
\text { Generalitat de Catalunya }\end{array}$ & $186.249,00 €$ \\
\hline $\begin{array}{l}\text { Apoyo a las personas } \\
\text { usuarias y sus } \\
\text { familias }\end{array}$ & $\mathrm{N}^{\circ}$ de asistentes a charlas & $\begin{array}{l}\text { Precio medio de conferencias de } 1 \\
\text { hora }\end{array}$ & $\begin{array}{l}\text { Consulta de precios de } \\
\text { actividades similares } \\
\text { (Diputació de Barcelona, } \\
\text { 2013) }\end{array}$ & $3.160,00 €$ \\
\hline $\begin{array}{l}\text { Conocimiento } \\
\text { y experiencia } \\
\text { profesional }\end{array}$ & $\begin{array}{l}\text { Coste de la estructura de } \\
\text { dedicación de CO y USAP }\end{array}$ & Coste del personal & $\begin{array}{l}\text { Estimación de La } \\
\text { Fundació Rubricatus }\end{array}$ & $153.336,18 €$ \\
\hline \multirow{2}{*}{$\begin{array}{l}\text { Beneficios para las } \\
\text { familias tutoras }\end{array}$} & $\begin{array}{l}\mathrm{N}^{\circ} \text { de usuarios/as y trabajadores/ } \\
\text { as dependientes de familiares } \\
\text { STO }\end{array}$ & $\begin{array}{l}\text { Ahorro del precio de asistencia a un } \\
\text { recurso de pago }\end{array}$ & $\begin{array}{l}\text { Consulta a residencia } \\
\text { privada que dispone del } \\
\text { recurso de CO }\end{array}$ & $809.004,00 €$ \\
\hline & $\begin{array}{l}\text { № de usuarios/as y trabajadores/ } \\
\text { as dependientes de familiares en } \\
\text { los que trabajan ambos miembros }\end{array}$ & $\begin{array}{l}\text { Salario que se ha podido mantener } \\
\text { por parte de las familias (salario } \\
\text { bruto medio } 2017 \text { en Cataluña) }\end{array}$ & $\begin{array}{l}\text { Instituto Nacional de } \\
\text { Estadística: Encuesta } \\
\text { anual de estructura } \\
\text { salarial } 2017\end{array}$ & $251.804,50 €$ \\
\hline \multirow{2}{*}{$\begin{array}{l}\text { Sensibilización de la } \\
\text { comunidad }\end{array}$} & $\begin{array}{l}\mathrm{N}^{\circ} \text { de alumnos que han asistido a } \\
\text { los talleres en las escuelas }\end{array}$ & $\begin{array}{l}\text { Precio estándar de mercado de } \\
\text { actividades y talleres infantiles y } \\
\text { familiares de } 1 \text { hora }\end{array}$ & $\begin{array}{l}\text { Precio medio de talleres } \\
\text { para niños y niñas (Roots } \\
\text { for Sustainability, 2019) }\end{array}$ & $7.518,00 €$ \\
\hline & $\begin{array}{l}N^{\circ} \text { de noticias en medios de } \\
\text { comunicación (TV, radio, prensa } \\
\text { escrita) }\end{array}$ & Tarifas periodísticas & $\begin{array}{l}\text { Col-legi de Periodistes de } \\
\text { Catalunya (2008) }\end{array}$ & $1.250,00 €$ \\
\hline \multirow{2}{*}{$\begin{array}{l}\text { Actividades de } \\
\text { voluntariado }\end{array}$} & $\begin{array}{l}N^{\circ} \text { de horas de voluntariado hacia } \\
\text { la fundación/de la fundación hacia } \\
\text { la comunidad }\end{array}$ & $\begin{array}{l}\text { Coste de oportunidad (salario medio } \\
\text { por hora en Cataluña sin cargas } \\
\text { impositivas) }\end{array}$ & $\begin{array}{l}\text { Instituto Nacional de } \\
\text { Estadística: Encuesta } \\
\text { anual de estructura } \\
\text { salarial } 2017\end{array}$ & $16.134,67 €$ \\
\hline & $\begin{array}{l}\mathrm{N}^{\circ} \text { de personas participantes en } \\
\text { "espigamientos" } 5\end{array}$ & $\begin{array}{l}\text { Precio por persona para actividades } \\
\text { de teambuilding }\end{array}$ & $\begin{array}{l}\text { Consulta de precios de } \\
\text { actividades outdoor para } \\
\text { empresas, Noviembre } \\
2019\end{array}$ & $8.100,00 €$ \\
\hline $\begin{array}{l}\text { Valor de proveedor } \\
\text { social }\end{array}$ & $\begin{array}{l}\text { Facturación de acuerdo con los } \\
\text { criterios de LISMI }\end{array}$ & $\begin{array}{l}\text { Facturación en concepto de } \\
\text { medidas alternativas de acuerdo } \\
\text { con la Ley General de Discapacidad } \\
\text { (LGD) }\end{array}$ & $\begin{array}{l}\text { Contabilidad de La } \\
\text { Fundació Rubricatus }\end{array}$ & $90.780,09 €$ \\
\hline $\begin{array}{l}\text { Confianza y } \\
\text { transparencia }\end{array}$ & Cesión de uso de espacios & $\begin{array}{l}\text { Valor de los equipamientos cedidos } \\
\text { por el Ayuntamiento }\end{array}$ & $\begin{array}{l}\text { Contabilidad de La } \\
\text { Fundació Rubricatus }\end{array}$ & $328.758,58 €$ \\
\hline
\end{tabular}

5. Acciones de recogida en el campo de frutas y verduras que son descartadas del circuito comercial organizadas por la entidad Espigoladors (http://www.espigoladors.cat). 


\begin{tabular}{|c|c|c|c|c|}
\hline Variable de valor & Indicador & Proxy & Fuente & $\begin{array}{c}\text { Valor } \\
\text { monetario } 2018\end{array}$ \\
\hline \multirow{2}{*}{$\begin{array}{l}\text { Impacto positivo en el } \\
\text { medio ambiente }\end{array}$} & $\begin{array}{l}\mathrm{N}^{\circ} \text { de contenedores recuperados } \\
\text { y que ahorran la compra de } \\
\text { nuevos contenedores }\end{array}$ & $\begin{array}{l}\text { Diferencia entre coste medio de } \\
\text { un contenedor nuevo y coste de la } \\
\text { reparación }\end{array}$ & $\begin{array}{l}\text { Estimación de La } \\
\text { Fundació Rubricatus }\end{array}$ & $176.400,00 €$ \\
\hline & $\begin{array}{l}\mathrm{N}^{\circ} \text { de horas de dedicación a } \\
\text { proyectos de economía circular }\end{array}$ & Coste/hora trabajador & $\begin{array}{l}\text { Estimación de La } \\
\text { Fundació Rubricatus }\end{array}$ & $5.760,00 €$ \\
\hline \multirow{2}{*}{$\begin{array}{l}\text { Capacitación para la } \\
\text { pre-empleabilidad }\end{array}$} & $N^{\circ}$ de horas de prácticas & $\begin{array}{l}\text { Coste/hora trabajador de categoría } \\
\text { equiparable }\end{array}$ & $\begin{array}{l}\text { Estimación de La } \\
\text { Fundació Rubricatus }\end{array}$ & $7.264,08 €$ \\
\hline & Plan de formación UNO a UNO & Dotación del programa formativo & $\begin{array}{l}\text { Contabilidad de La } \\
\text { Fundació Rubricatus }\end{array}$ & $24.932,20 €$ \\
\hline \multirow[b]{2}{*}{$\begin{array}{l}\text { Formación a los } \\
\text { trabajadores/as }\end{array}$} & $\mathrm{N}^{\circ}$ de horas de formación interna & $\begin{array}{l}\text { Máximo coste/hora de formación } \\
\text { presencial superior bonificada }\end{array}$ & $\begin{array}{l}\text { Datos de la Fundación } \\
\text { Estatal para la Formación } \\
\text { en el Empleo (FUNDAE) }\end{array}$ & $14.599,00 €$ \\
\hline & $\begin{array}{l}N^{\circ} \text { de horas de formación interna } \\
\text { profesionalizadora }\end{array}$ & $\begin{array}{l}\text { Importes de los módulos } \\
\text { económicos por alumno y hora } \\
\text { adaptados a personas con } \\
\text { discapacidad (rango superior) }\end{array}$ & $\begin{array}{l}\text { Servicio Público } \\
\text { de Empleo de } \\
\text { Cataluña: Resolución } \\
\text { TSF/1892/2018, de } 1 \text { de } \\
\text { agosto }\end{array}$ & $2.025,00 €$ \\
\hline \multicolumn{4}{|l|}{ TOTAL } & $2.552 .568,72 €$ \\
\hline
\end{tabular}

Fuente: elaboración propia.

\subsection{Cálculo del Valor Social Integrado}

EI Valor Social Integrado (VSI) corresponde al valor social consolidado, el cual — similar al concepto contable de la misma denominación - tiene en consideración el valor conjunto generado, evitando duplicar el valor que se genera a la vez para varios stakeholders o varias tipologías de valor. En este sentido, se hace la suma del valor económico social (VES) y el valor social específico (VASE), pero restando todas las partidas de este último que coinciden con ingresos o gastos efectuados y destinados a beneficiar a un stakeholder determinado ${ }^{6}$. En consecuencia, se obtiene como resultado del año 2018 un VSI consolidado de 7.298.116€. Aparte del valor económico social (VES) y el valor social específico (VASE), es interesante analizar también el retorno económico a las Administraciones públicas derivado de los ingresos impositivos directos e indirectos de la actividad económica y los ahorros de prestaciones sociales (Huete y Quezada, 2014), y que para el año 2018 asciende a $1.014 .962 €$.

En la figura 4 se presentan de forma gráfica las tipologías de valor social en círculos de menor a mayor tamaño. Mientras que el resultado económico considera solamente el excedente económico obtenido por la organización, el retorno a las Administraciones públicas contabiliza el impacto de la actividad para el erario. Tomando una visión más amplia, el valor económico social (VES) y el valor social específico (VASE)

6. En el caso de La Fundació Rubricatus se resta la facturación en concepto de medidas alternativas de acuerdo con la LGD que expresa el valor de la fundación como proveedor social, debido a que ya está considerada en el valor distribuido a clientes (facturación). 
contemplan el valor generado por la organización para sus diferentes grupos de interés, dentro y fuera de las relaciones de mercado. Finalmente, el Valor Social Integrado (VSI) representa la consolidación de VES y VASE para el conjunto de grupos de interés.

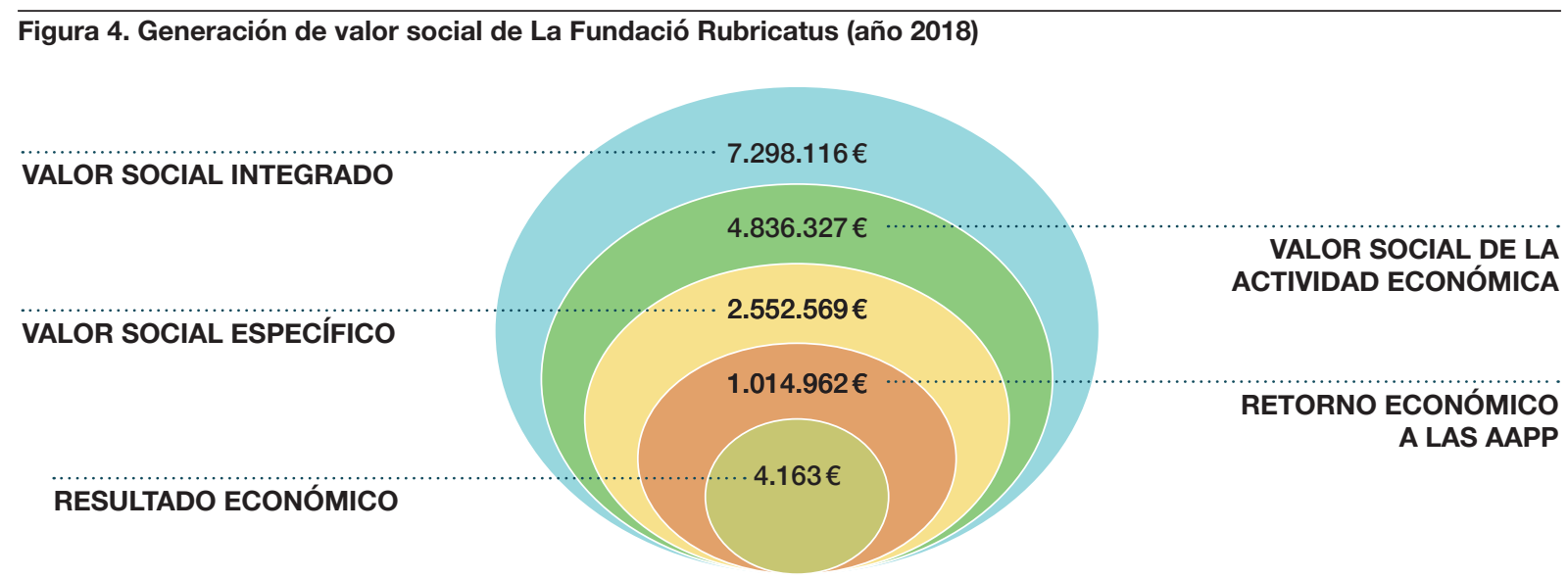

Fuente: elaboración propia.

La tabla 8 muestra la distribución del valor social generado por La Fundació Rubricatus a los diferentes grupos de interés. Las personas trabajadoras, las Administraciones públicas y los clientes son los stakeholders que más valor reciben. Mientras estos colectivos reciben su mayor aportación en forma de valor de mercado; las familias, la ciudadanía y los usuarios/as son los stakeholders que se benefician exclusivamente de un valor social al margen de las relaciones de mercado. Debido a que no se ha podido contabilizar la mejora emocional y cognitiva de las personas usuarias, el valor social de éstos resulta relativamente bajo en comparación con los otros grupos de interés.

Tabla 8. Distribución del valor social por grupos de interés (año 2018)
\begin{tabular}{|l|c|c|c|}
\hline & Valor económico social (VES) & Valor social específico (VASE) & Valor total por stakeholder \\
\hline AP & $1.348 .468,46 €^{\star}$ & $470.524 €$ & $\mathbf{1 . 8 1 8 . 9 9 2 €}$ \\
\hline Trabajadores/as & $2.136 .757,71 €^{\star \star}$ & $584.595 €$ & $\mathbf{2 . 7 2 1 . 3 5 3 €}$ \\
\hline Proveedores & $120.693,82 €$ & - & $\mathbf{1 2 0 . 6 9 4 €}$ \\
\hline Clientes & $1.155 .708,46 €^{\star \star \star}$ & $90.780 €$ & $\mathbf{1 . 2 4 6 . 4 8 9 €}$ \\
\hline Usuarios/as & & $78.253 €$ & $\mathbf{7 8 . 2 5 3 €}$ \\
\hline Familias & & $1.164 .426 €$ & $\mathbf{1 . 1 6 4 . 4 2 6 €}$ \\
\hline Estudiantes & & $32.196 €$ & $\mathbf{3 2 . 1 9 6 €}$ \\
\hline Voluntarios/as & & $13.611 €$ & $\mathbf{1 3 . 6 1 1 €}$ \\
\hline Ciudadanía & & 530.311 & $\mathbf{5 3 0 . 3 1 1 €}$ \\
\hline
\end{tabular}

* incluye facturación a clientes públicos; **incluye personas trabajadoras con y sin discapacidad; ***se refiere a facturación a clientes privados Fuente: elaboración propia. 
En la figura 5 se representa gráficamente el valor social generado por La Fundació Rubricatus a los grupos de interés en las diferentes tipologías de valor. Como se puede observar, la suma de todos los valores generados para los stakeholders $(7.726 \mathrm{k} €$ ) excede el importe del VSI consolidado $(7.298 \mathrm{k} €)$. Esta diferencia representa el valor compartido generado, es decir, el valor que se genera de forma simultánea para más de un stakeholder, pero que sólo se contabiliza una vez en el importe del VSI.

En este sentido, se han identificado variables de valor que coinciden parcial o totalmente entre diversos grupos de interés. Un ejemplo es el conocimiento y experiencia profesional en los servicios prestados de los que se benefician tanto los trabajadores/as, usuarios/as y familiares, como las Administraciones públicas -en el caso de que no existiera la fundación, tendrían que crear una estructura que diese cobertura a las personas discapacitadas del municipio. Otro ejemplo son las actividades de voluntariado que aportan gratificación a las personas voluntarias y, al mismo tiempo consiguen un impacto positivo en personas trabajadoras, usuarias o en el entorno social y/o ambiental.

\section{Figura 5. Valor social generado a los grupos de interés de La Fundació Rubricatus (año 2018)}

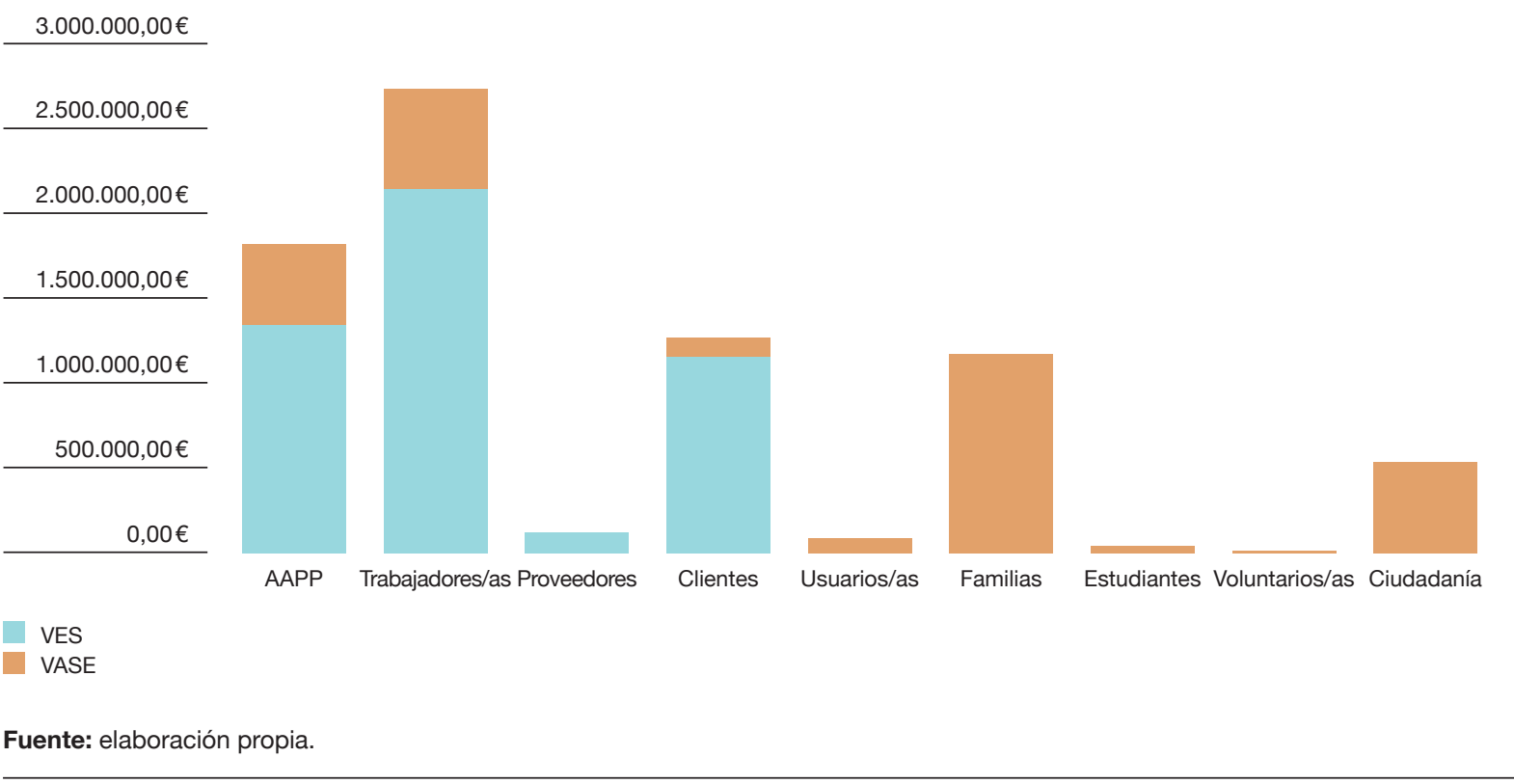

Aparte de los resultados del valor social generado en términos absolutos, es interesante considerar la rentabilidad social de la actividad de La Fundació Rubricatus mediante diferentes ratios de análisis. Con respecto al presupuesto de la fundación del año 2018 (3.253.287€), proveniente de la facturación pública y privada, las subvenciones recibidas de las Administraciones públicas y las donaciones privadas; por cada euro de este presupuesto se generan 2,24 euros de Valor Social Integrado.

En la figura 6 se muestra cómo se distribuye este valor a los principales grupos de interés según el formato poliédrico de análisis (juntando los grupos de interés de personas usuarias y familias). En consonancia con 
la distribución de valor descrita antes, se observa que el mayor retorno es para las personas trabajadoras, seguidas de las Administraciones públicas, los clientes y las familias/personas usuarias, la ciudadanía y los proveedores.

Figura 6. Retorno de valor social para los grupos de interés de La Fundació Rubricatus (año 2018)

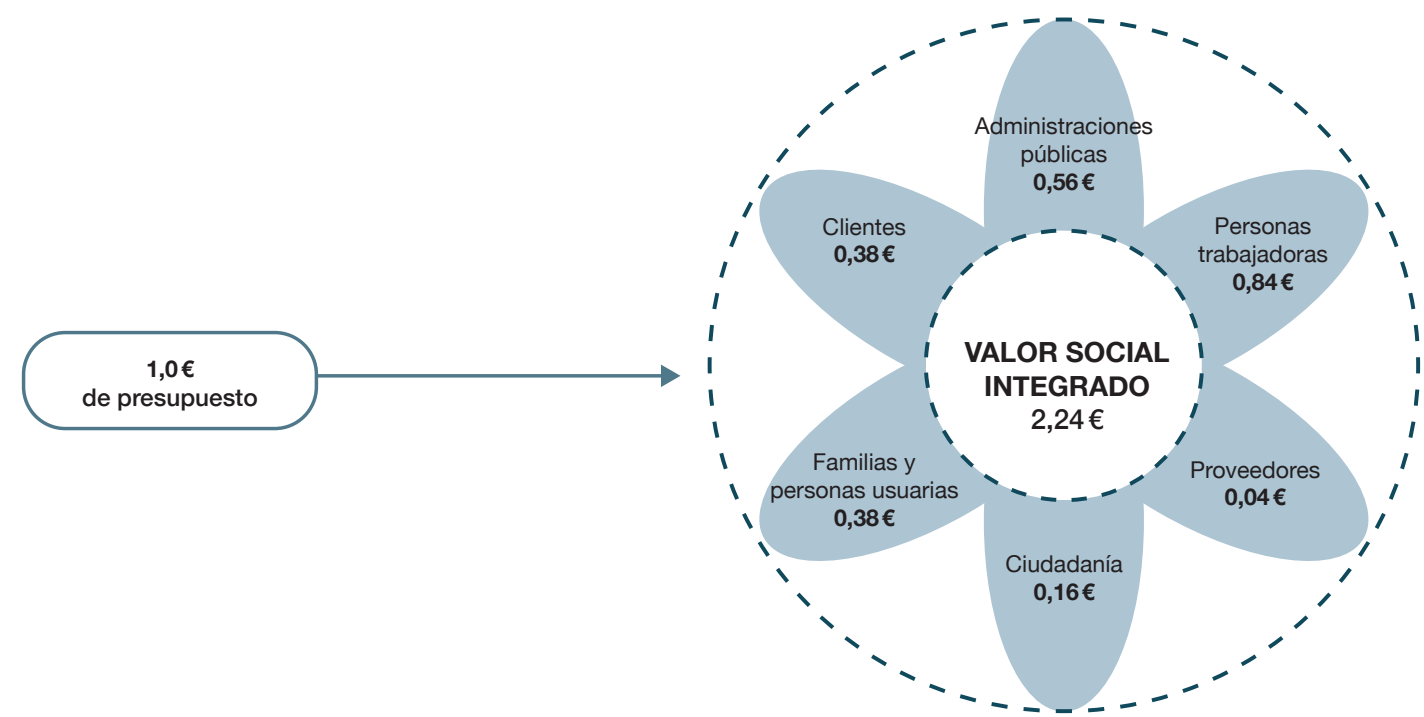

Fuente: elaboración propia.

Asimismo, se pueden relacionar los resultados del cálculo con la financiación pública recibida, para calcular el retorno de la inversión pública. La financiación pública de La Fundació Rubricatus en el año 2018 supuso 1.428.915€. Como consecuencia, por cada euro de gasto público destinado a la actividad de La Fundació Rubricatus, se retornan 5,11 euros a diferentes grupos de interés de la sociedad; es decir, se genera un valor de más de cinco veces la financiación pública recibida.

Es importante destacar que los resultados de valor social y las correspondientes ratios serían más elevados si se hubiera incluido el valor social generado por las mejoras en el bienestar de las personas con discapacidad (trabajadoras y usuarias). Por ejemplo, un estudio de SROI aproxima el valor monetario anual de las mejoras en la calidad de vida de un usuario de un centro ocupacional en $5.225 €$, basándose en la reducción de las necesidades de apoyo y el correspondiente salario/hora de un auxiliar de ayuda a domicilio (ECODES y Alter Civites, 2013). Otro estudio recurre a análisis de "precios sombra" relacionados con diferentes dimensiones de satisfacción con la vida para calcular las mejoras en bienestar, autonomía y relaciones sociales de un trabajador con discapacidad de un CEE en 7.101€ al año (ECODES y Alter Civites, 2018). Otros trabajos incluso cifran las mejoras experimentadas por personas con diversidad funcional usuarias de un servicio de asistencia personal a través de los precios de servicios equivalentes en el mercado privado (asistente personal, auxiliar del hogar, tratamiento psicológico, socio en un club, etc.), y llegan a importes por usuario de 32.247€ (láñez et al., 2018) y 44.644€ (Ayuntamiento de Barcelona, 2012) 7 .

7. Debido a que los períodos de análisis de los citados estudios abarcan más de un año, las cifras se han corregido con los factores correspondientes. 
Asimismo, estos estudios monetizan las mejoras en la calidad de vida del entorno cuidador, generalmente las familias de las personas discapacitadas. Desde un enfoque conservador, si en el caso de La Fundació Rubricatus monetizáramos el valor social vinculado a las mejoras en bienestar en $5.000 €$ por trabajador y usuario, se obtendría un VSI consolidado de 8.163k€, un retorno de 2,51 euros por cada euro de presupuesto y un retorno de 5,71 euros por cada euro de financiación pública.

\section{Conclusiones}

El objetivo del presente estudio era analizar y calcular el Valor Social Integrado (VSI) generado por las actividades realizadas por La Fundació Rubricatus para promover la calidad de vida de las personas con discapacidad intelectual. Esta metodología ha permitido cuantificar en términos monetarios el impacto económico y social creado. El análisis ha seguido un proceso de investigación-acción participativa con representantes de la entidad y sus grupos de interés (personas trabajadoras y usuarias con discapacidad, sus familias, Administraciones públicas, etc.), lo que ha permitido identificar de manera sistemática las dimensiones o variables de valor percibidas como relevantes.

Se ha calculado el VSI, que incluye tanto el valor social generado a través de la actividad económica - efecto de mercado capturado por indicadores de contabilidad- como el valor social específico generado a los diferentes stakeholders mediante relaciones ajenas a las transacciones de mercado - efecto de no mercado monetizado con ayuda de los indicadores y proxies utilizados-. El denominado valor social específico integra el conjunto de las variables de valor identificadas en la consulta con los stakeholders y visibiliza los beneficios experimentados sobre todo por las personas trabajadoras con discapacidad, personas usuarias, familias y ciudadanía.

Aunque se trata de una primera aproximación del cálculo del VSI, el estudio de caso muestra el interés de la contabilidad social para las entidades de la economía social como La Fundació Rubricatus, entidades que asumen un papel principal en la integración sociolaboral de las personas con discapacidad y que se caracterizan por la generación de valor social por encima de los resultados económicos o financieros (Fundación ONCE, 2015). La posibilidad de utilizar este método para evaluar su impacto social les permite a estas entidades demostrar de forma monetaria su valor social añadido, y mejorar de esta manera su competitividad frente a las empresas mercantiles.

En este sentido, cuantificar el valor social desde una perspectiva de contabilidad social tiene varias implicaciones prácticas para las empresas sociales. Por un lado, el proceso de medición del valor social proporciona una manera de atender las demandas crecientes de mayor responsabilidad y transparencia en la comunicación con los diferentes grupos de interés. En particular, la experiencia muestra que se produce una mejora en la relación con las personas empleadas, que una vez que conocen el resultado del valor social que generan sus organizaciones, se sienten orgullosas y más comprometidas con la empresa (Lazkano y Beraza, 2019). Asimismo, la contabilidad del valor social también puede ser útil en la relación con las Administraciones públicas que subvencionan las entidades que trabajan para la integración de personas con 
discapacidad, y ayudar a evaluar la eficacia y eficiencia de los recursos públicos destinados (Ruiz-Lozano et al., 2020).

Por otro lado, cuantificar el valor social de las empresas sociales puede ayudar a transformar la contabilidad financiera convencional y mejorar la estrategia y la gestión interna de las organizaciones de acuerdo con principios de ética y sostenibilidad. De esta manera, el VSI puede representar un instrumento eficaz de control interno, ya que puede dar información sobre posibles ineficiencias en el alcance de la misión social y mostrar potencial para la mejora de las prácticas de gestión. En concreto, pueden derivarse diferentes ratios de análisis, cuyo seguimiento y evaluación ayudarán en los procesos de toma de decisión y mejorarán el sistema de gestión organizativo (Ruiz-Lozano et al., 2020).

La investigación futura debería abordar las limitaciones actuales del cálculo del VSI, que como en otras metodologías de medición de impacto social están relacionadas con la disponibilidad de los datos necesarios para la cuantificación y monetización. A pesar de que la metodología del VSI reduce el esfuerzo requerido en la recogida de datos en comparación con el SROI - no aspira a medir el impacto final, sino que se conforma con indicadores y proxies intermedios - sería interesante establecer la relación de la actividad de la organización con las variables de valor intangibles como las mejoras cognitivas y emocionales alcanzadas en las personas con discapacidad. A medida de que se extienda la contabilidad social a más entidades que trabajan para la integración de personas con discapacidad, será más fácil consensuar métricas consistentes de valor social tangible e intangible y realizar procesos de comparación o benchmarking. 


\section{Referencias bibliográficas}

Ayuntamiento de Barcelona (2012). Evaluación del impacto social del Servicio de Asistente Personal del Instituto Municipal de Personas con Discapacidad. Barcelona: Área de Calidad de Vida, Igualdad y Deportes, Ayuntamiento de Barcelona.

Ayuso, S. (septiembre, 2020). "El valor social generado por un centro especial de empleo: comparación del análisis SROI y VSI”. Comunicación presentada en el XVIII Congreso Internacional de Investigadores en Economía Social y Cooperativa CIRIEC, Mataró.

Cataluña. Orden TSF/91/2017, de 15 de mayo, por la que se aprueban las bases reguladoras para la concesión de subvenciones a los centros especiales de empleo. Diario Oficial de Cataluña,19 de mayo de 2017, núm. 7373, pp. 1-23

Cataluña. Resolución TSF/1892/2018, de 1 de agosto, por la que se establecen los módulos económicos aplicables a las acciones de formación profesional para el empleo, destinadas prioritariamente a personas trabajadoras desempleadas, que promueve el Servicio Público de Empleo de Cataluña, Diario Oficial de Cataluña, 7 de agosto de 2018, núm. 7680, pp. 1-4.

Col·legi de Periodistes de Catalunya (2008). Tarifes mínimes orientatives per a l'exercici professional periodístic 2008. Recuperado de https://www.estrategiadelcontenido.com/doc/nots_28.pdf.

Diputació de Barcelona (2013). El retorn a la inversió de la Xarxa de Biblioteques Municipals de la província de Barcelona (2007-2011). Barcelona: Diputació de Barcelona.

ECODES y Alter Civites (2013). Análisis del retorno económico y social de la inversión en el Centro Ocupacional Comarcal de Sobrarbe en Boltaña de Atades Huesca, mediante aplicación de la metodología SROI (Retorno Social de las Inversiones). Informe de resultados. Huesca: Atades.

ECODES y Alter Civites (2018). Análisis del retorno social y socioeconómico de ATADI Empleo mediante la aplicación de la metodología SROI (Retorno Social de las Inversiones). Informe de resultados. Zaragoza: Plena Inclusión Aragón/ATADI.

Elkington, J. (1997). Cannibals with Forks: The Triple Bottom Line of 21st Century Business. Oxford: Capstone.

Emerson, J. et al. (2003). The Blended Value Map: Tracking the Intersects and Opportunities of Economic, Social and Environmental Value Creation. Menlo Park: William and Flora Hewlett Foundation.

Freeman, R. E. (1984). Strategic Management: A Stakeholder Approach. Boston: Pitman.

Freeman, R. E. et al. (2010). Stakeholder Theory: The State of the Art. Cambridge: Cambridge University Press.

Fundación ONCE (2015). Las empresas de la economía social como palanca de creación de empleo para las personas con discapacidad. Recuperado de https://bit.ly/37t7Mv5.

Huete, A. y Quezada, M. Y. (2014). “Análisis de Retorno Social de la Inversión en dos sistemas de apoyo a personas con gran discapacidad: asistencia personal y servicio residencial. Un estudio de caso". Revista Española de Discapacidad, 2(1), pp. 51-69.

láñez, A. et al. (2018). "Impacto económico y social de la asistencia personal a través de la metodología del Retorno Social de la Inversión". Revista Española de Discapacidad, 6(2), pp. 81-102. 
Instituto Nacional de Estadística (2019). Encuesta anual de estructura salarial 2017. Recuperado de https://bit. ly/37rZB25.

Jordán de Uríes, F. B. et al. (2014). "Aproximación al análisis coste-beneficio entre empleo con apoyo y centros especiales de empleo mediante simulación comparativa con 24 trabajadores". Revista Española de Discapacidad, 2(1), pp. 33-50.

Lazkano, L. y Beraza, A. (2019). "Social accounting for sustainability: A study in the social economy". Sustainability, 11(24), pp. 6894.

Maier, F. et al. (2015). "SROI as a method for evaluation research: Understanding merits and limitations". Voluntas: International Journal of Voluntary and Nonprofit Organizations, 26(5), pp. 1805-1830.

Porter, M. E. y Kramer, M.R. (2011). "Creating shared value”. Harvard Business Review, 89(1/2), pp. 62-77.

Retolaza, J. L. (2012). Análisis coste-beneficio de las empresas de inserción: elaboración de un modelo de análisis costo-beneficio. Madrid: Editorial Académica Española.

Retolaza, J. L. et al. (2014). "Cuantificación del valor social: propuesta metodológica y aplicación al caso de Lantegi Batuak”. Zerbitzuan: Gizartezerbitzuetarakoaldizkaria (Revista de Servicios Sociales), 55, pp. 17-33.

Retolaza, J. L. et al. (2016). Social Accounting for Sustainability: Monetizing the Social Value. Berlin/Heidelberg: Springer.

Retolaza, J. L. y San-José, L. (2018). "Contabilidad social para el bien común”. Revista de Responsabilidad Social de la Empresa, 29, pp. 95-122.

Rodríguez, G. et al. (2015). Los beneficios de la inclusión social de las personas con discapacidad. Madrid: Grupo Editorial Cinca.

Roots for Sustainability (2019). El relato de la acción en sostenibilidad de Danone España (no publicado).

Ruiz-Lozano, M. et al. (2020). "SROI methodology for public administration decisions about financing with social criteria. A case study". Sustainability, 12(3), pp. 1070.

Social Value International (2019). What is Social Value? Recuperado de https://socialvalueint.org/social-value/ what-is-social-value/. 